\title{
BEHINDERTENHILFE
}

\section{Die Chancen ergreifen}

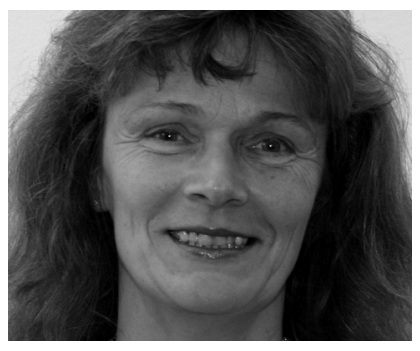

VON CLAUDIA ZINKE

Claudia Zinke ist Referentin für Teilhabe und Gesundheit beim Paritätischen Wohlfahrtsverband - Gesamtverband e. V. E-Mail

Claudia.Zinke@paritaet.org
Die Politik für Menschen mit Behinderung steht vor großen Herausforderungen. Insbesondere die Umsetzung der Behindertenkonvention der Vereinten Nationen und die Reform der Eingliederungshilfe fordern dabei die Wachsamkeit der Wohlfahrtsverbände und die Innovationskraft der Leistungserbringer.

Die Rahmenbedingungen sollen für Menschen mit und ohne Behinderungen positiv gestaltet werden. Voraussetzung hierfür ist u. a. Barrierefreiheit in allen Bereichen. Politische Entscheidungen müssen sich an den Inhalten UN-Konvention über die Rechte der Menschen mit Behinderungen messen lassen. Es soll ein Aktionsplan zur Umsetzung der UN-Behindertenrechtskonvention erstellt werden - so steht es geschrieben im Koalitionsvertrag der Regierung zwischen CDU, CSU und FDP vom Oktober 2009 für die 17. Legislaturperiode.

Grundsätzlich können und müssen diese Zeilen optimistisch verstanden werden. Sie könnten eine Chance für die künftige Ausgestaltung der Hilfen und Angebote für Menschen mit Behinderungen bieten, wenn sich die Leitgedanken der Behindertenrechtskonvention wie beispielsweise einem neuen Verständnis von Behinderung, Inklusion, unabhängige Lebensführung, Würdigung der menschlichen Vielfalt, Gleichstellung und Empowerment in der Behindertenpolitik in Deutschland widerspiegeln.

Welche konkreten Vorhaben sich hinter den Zeilen im Koalitionsvertrag verbergen und wie diese Ziele umgesetzt werden, wird sich voraussichtlich im Laufe dieses Jahres abzeichnen. Dann werden die Diskussionen zu konkreten Vorschlägen für einen Nationalen Aktionsplan und zu den Überlegungen in den verschiedenen Bereichen der Sozialleistungen begonnen oder fortgesetzt. Dazu gehören vor allem die Vorschläge der Arbeits- und Sozialministerkonferenz zur Weiterentwicklung der Eingliederungshilfe für Menschen mit Behinderungen. Aber auch der Vorschlag für ein neues Begutachtungsinstrument in der Pflegeversicherung für Menschen mit Pflegebedarf sowie die Bestrebung, die Schnittstellen zwischen Jugendhilfe und der Sozialhilfe abzubauen und Freiwilligendienste auszubauen, haben Bedeutung für Menschen mit Behinderungen. Darüber hinaus werden die Krankenversicherung und die Bereiche Gesundheit und Arbeit im Koalitionsvertrag thematisiert. All diese Vorhaben sind mit Blick auf die Entwicklung eines Nationalen Aktionsplans zur Umsetzung der UN-Behindertenrechtskonvention kritisch zu begleiten und einer Prüfung bezüglich der Leitgedanken der UN-Behindertenrechtskonvention zu unterziehen.

\section{Blick zurück}

Bei so vielen Prüfungs- und Entwicklungsvorhaben der Bundesregierung lohnt ein Rückblick, um die Möglichkeiten der Veränderungen realistischer betrachten $\mathrm{zu}$ können. In der Vergangenheit hatte man sich schon vieles vorgenommen, beispielsweise in der letzten Legislaturperiode »die Leistungsstrukturen der Eingliederungshilfe weiterzuentwickeln « oder »die Verzahnung ambulanter und stationärer Dienste « (Quelle Koalitionsvertrag 16. Legislaturperiode).

Jedoch scheiterte dies an unterschiedlichen Faktoren, zum Beispiel an der Prioritätensetzung der Regierungsaufgaben. Die Eingliederungshilfe für Menschen mit Behinderungen ist neben der Bankenkrise, den Umweltthemen oder den Steuerentlastungen für Wirtschaft und Bürgerinnen und Bürger öffentlichkeitswirksam eher marginal, auch wenn 713.000 Menschen mit Behinderungen Leistungen der Eingliederungshilfe erhalten. Die Ausgaben der Eingliederungshilfe von 11,2 Milliarden Euro (Angaben laut Statistischem Bundesamt) sind ein großer Posten in den Haushalten der Länder und Kommunen. Die Kommunen melden sich bereits mit Schlagzeilen zu Wort, wie »Sozialausgaben entwickeln immer größere Sprengkraft für die Kommunalhaushalte - Städte brauchen Entlastung " und gehen in ihren Beiträgen auf die Zunahme der Empfänger von Sozialleistungen ein.

Im Verhältnis zu den von der Bundesregierung im Jahr 2008 beschlossenen Rettungspaket für die Banken von 500 Milliarden Euro oder im Verhältnis zu den 30 
Milliarden Euro, die schätzungsweise Jahr für Jahr verschwendet werden (Quelle: Bund der Steuerzahler Deutschland e.V.) erscheinen 11,2 Milliarden Euro jedoch in einem anderen Licht. Wenn Bund, Länder und Gemeinden auch in anderen Bereichen weniger großzügig und dafür effizienter mit dem Geld der Steuerzahler umgehen würden, könnten mühelos zweistellige Milliardenbeträge eingespart und den sozialen Systemen zur Verfügung stehen. Auch 2009 verteilte die Bundesregierung weitere Geldgeschenke. Dazu gehörten u. a. das Konjunkturpaket, wieder die Bankenrettung, Abwrackprämie, Kinderbonus, Bauaufträge und Abgabensenkung. Bei der Umverteilung dieser Lasten zwischen dem Bund, den Ländern und Kommunen wird das letzte Glied in der Kette die Auswirkungen am stärksten zu spüren bekommen. Das sind Empfänger von Sozialleistungen wie Arbeitslosengeld und Eingliederungshilfe, arbeitslose Menschen und insbesondere deren Kinder oder Menschen, die behindert und oder chronisch krank sind. Menschen, die aufgrund ihrer persönlichen Lebensumstände auf Unterstützung der Gesellschaft angewiesen sind und die einen Rechtsanspruch auf bedarfsdeckende Leistungen haben.

Darüber hinaus wird verkannt, dass ein Großteil der Ausgaben der Eingliederungshilfe für Menschen mit Behinderungen u. a. deshalb notwendig ist, weil die regionalen Bedingungen kaum bis gar nicht barrierefrei sind. Wären beispielsweise alle öffentlichen Einrichtungen, einschließlich der öffentlichen Verkehrsmittel barrierefrei gestaltet, könnte ein Teil der notwendigen Mittel für persönliche Assistenz oder Mobilitätshilfen eingespart werden. Unzureichend im Blick ist die Verantwortung der Kommunen und Gemeinden für Menschen mit Behinderungen in anderen Bereichen, wie dem Bauen, der Wirtschaft oder dem Verkehr. So wurde beispielsweise 2006 beim Theaterneubau Potsdam die DINNorm nicht eingehalten und der Einbau behindertengerechter Toiletten vergessen. Nachträglich mussten diese mit einem Extra-Aufwand eingebaut werden.

Vom Bund ist kaum Entlastung zu erwarten. So fehlte beispielsweise beim Konjunkturpaket der Bundesregierung und den damit verbundenen Bauaufträgen die Verpflichtung zur Barrierefreiheit. Ein Leistungsgesetz für Menschen mit Behinderungen oder die Beteiligung des Bundes an den Kosten der Eingliederungshilfe wird vonseiten der Bundesregierung abgelehnt. Infolge dessen wird insbesondere auf der kommunalen Ebene überlegt, wie an den Sozialhilfeausgeben insbesondere den Leistungen der Eingliederungshilfe für Menschen mit Behinderungen gespart werden kann. Die Zauberformel heißt: planen und steuern.
Dabei ist wahrnehmbar, dass mindestens auf zwei Ebenen geplant und gesteuert wird - auf der Individualebene beim konkreten Unterstützungsbedarf des Leistungsberechtigten und auf der Strukturebene bei der Angebotsform und der Vergütung der Sozialleistungen. Grundsätzlich spricht nichts gegen Planung, Steuerung und sparsamer und wirtschaftlicher Erbringung der Leistungen, wenn dieses ressortübergreifend erfolgt und, wenn Planung und Steuerung der individuellen Unterstützungsleistungen und Unterstützungsangebote ausschließlich den Bedarfen und Rechtsansprüchen der Leistungsberechtigten verpflichtet sind. Allerdings ist zunehmend wahrnehmbar, dass ausschließlich die Finanzlage der jeweiligen Kommunen oder Länder die Grundlage des Handelns bildet.

Natürlich ist die Politik des »Um- und Verteilens « bei den sozialen Sicherungsleistungen zwischen den verschiedenen Interessenverbänden, beispielsweise der Menschen mit Behinderungen, der Einrichtungsvertretungen und der öffentlichen Verwaltung immer strittig gewesen. Aber die Möglichkeit, einen Konsens zwischen den verschiedenen Gruppen zu erreichen, wird mit der zunehmenden Ökonomisierung der Sozialpolitik und dem Spar-Wettlauf immer schwieriger. Im Folgenden sollen ausgewählte Beispiele der bisherigen und möglichen Veränderungen aufgeführt werden, in denen fachlich und fiskalisch sowohl Chancen als auch negative Entwicklungsmöglichkeiten liegen.

\section{Kommunalisierung: § 97 SGB XII Sachliche Zuständigkeit}

Mit der Änderung des $\$ 97$ Abs. 3 SGB XII wurden auf der bundesgesetzlichen Ebene neue Bestimmungen der sachlichen Zuständigkeit der Träger der Sozialhilfe wirksam. Danach ist der örtliche Träger der Sozialhilfe sachlich zuständig soweit nicht der überörtliche Sozialhilfeträger sachlich zuständig ist. Die sachliche Zuständigkeit des überörtlichen Sozialhilfeträgers wird

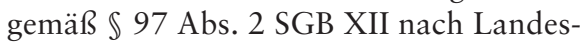
recht bestimmt.

Seit dem In-Kraft-Treten des $\$ 97$ (3) SGB XII im Jahr 2007 haben bisher fünf Bundesländer vom Landesrecht Gebrauch gemacht und die Kommunalisierung der Leistungen der Eingliederungshilfe umgesetzt, nämlich Thüringen, Brandenburg, Baden-Württemberg, Mecklenburg-Vorpommern und Schleswig-Holstein. Weitere Länder planen die Verlagerung der Verantwortung der Eingliederungshilfe auf die kommunale Ebene oder befinden sich in der modellhaften Erprobung mit ausgewählten Kommunen.

Leider produzieren diese Veränderungen unangenehme Schlagzeilen und lassen erahnen, in welche Richtung der Prozess gehen kann. Am 21. Dezember 2009 war in der Tageszeitung »taz « unter der Überschrift "Weniger Hilfe für Behinderte " zu lesen: "Schulden-Staat: Kreise kündigen Vertrag über Eingliederungshilfe - gegen den Willen von Land und Städten. Kommunen versuchen verzweifelt, Steuergeschenke von Schwarz-Gelb im Bund zu kompensieren. «In dem Artikel wird, wie bereits ausgeführt, über steigende Zahlen der Leistungsberechtigten und über die Sorge der Verbände hinsichtlich eines »Streichkonzertes « zulasten der Menschen mit Behinderungen und zulasten der Mitarbeitenden im Bereich der Betreuung und Förderung berichtet. Der Landrat räumt in dem Artikel ein, dass es bei den Sparvorhaben um "richtig große Summen " geht. Die Städte dagegen sorgen sich um ihren Ruf. Sie ließen mitteilen: »Leider wird an keiner Stelle aufgegriffen, dass die Sozialhilfeträger auch Verantwortung für die Menschen mit Behinderung tragen. Es wird ein Leichtes sein, uns als >Geldverwalter $<$ und 'Sparkommissare bloßzustellen. «

Bleibt zu hoffen, dass sie die Chance nutzen und nicht nur eine Streichliste pflegen. Dazu gehört eine mit dem öffentlichen Dienst vergleichbar tarifliche Entlohnung für Personen, die in diesem Bereich tätig sind. Vergütungen, die eine Sozialabsicherung dieser Personen nicht ermöglichen, wie beispielsweise Angebote von 4,50 Euro die Stunde, produzieren neue Abhängigkeit von Sozialtransferleistungen und dies nicht erst im Rentenalter.

Bleibt weiterhin zu hoffen, dass sie tatsächlich Planungs- und Steuerungsverantwortung bei der Schaffung von ambulanten und niederschwelligen Unterstützungsleistungen wahrnehmen. Die Interessenverbände behinderter Menschen und die Verbände der Freien Wohlfahrtspflege fordern seit Jahren eine am Gemeinwesen orientierte Politik und die Übernahme der Verantwortung für Menschen mit Behinderungen als Bürgerinnen und Bürger der Städte, Landkreise und Gemeinden.

Auf der Basis der Grundgedanken der Behindertenrechtskonvention wie Inklusion und Selbstbestimmung könnten die Kommunen unter Einbeziehung von professionellen Angeboten und bürgerschaftlichem Engagement einen Sozialraum gestalten, der allen und insbesondere den Bürgerinnen und Bürgern mit Behinderungen verpflichtet ist.

\section{Ambulante Unterstützungsleistungen}

Ambulante Unterstützungsleistungen sind eine wesentliche Voraussetzung, wenn der Sozialraum entsprechend den Bedürfnissen der Menschen und somit inklusiv ge- 
staltet werden soll. Neben den dargestellten fachlichen Überlegungen ist beispielsweise die Bundesarbeitsgemeinschaft der überörtlichen Sozialhilfeträger in einer Stellungnahme zu den fiskalischen Auswirkungen der Ambulantisierung in der Eingliederungshilfe zu dem Ergebnis gekommen, dass »bei allen untersuchten Berechnungen und Vergleichen in der Tendenz festgehalten werden kann, dass ambulante Betreuungsleistungen in der Regel für den zuständigen Träger der Eingliederungshilfe kostengünstiger erbracht werden können. Dieser Einspareffekt verringert sich zwar bei einer Nettogesamtbetrachtung aller Sozialhilfeaufwendungen, bleibt aber deutlich spürbar. Auch die Gesamtbetrachtung der Kosten der Sozialhilfeträger, also der Kosten der Eingliederungshilfe einschließlich der notwendigen den Lebensunterhalt sichernden Kosten führt oftmals zu dem gleichen Ergebnis. «

Eine Aussage, wenn auch von fiskalischen Aspekten geprägt, die Anlass sein sollte, ambulanten Leistungen zu fördern und auszubauen. Der Vorteil liegt auf der Hand, ein Leistungsmix wäre möglich, beispielsweise bestehend aus den Leistungen der Eingliederungshilfe nach SGB XII, den Leistungen der Pflegeversicherung nach SGB XI oder den Leistungen der Krankenversicherung wie der ambulanten Behandlungspflege.

Leider wird über die Leistung der ambulanten Behandlungspflege und deren »Orte ", an denen diese Leistung für Menschen mit Behinderungen erbracht werden kann, immer noch an vielen Stellen in der Bundesrepublik trotz der Richtlinien des Gemeinsamen Bundesausschusses gestritten. Oder es werden Voraussetzungen insbesondere bei der Qualifikation des Personals wie beispielsweise in der häuslichen psychiatrischen Krankenpflege oder Soziotherapie formuliert, die eine Anerkennung der bisherigen ambulanten Dienstleister für psychisch kranke Menschen faktisch unmöglich machen.

Neben den konkreten Dienstleistungen der Eingliederungshilfe, Pflege oder Behandlung sind auch notwendige Beratungsleistungen in den Unterstützungsangeboten und somit entsprechend im Budget der Leistungsbewilligung zu berücksichtigen. Voraussetzung für die Umsetzung eines ambulanten Leistungsmix sind Beratungs- und Unterstützungsleistungen. Vorstellbar wäre, eine Mindestmenge an Beratungsleistungen für Menschen mit Behinderungen in Form von Zeiteinheiten oder eines Geldbetrages pauschal zu gewähren.

Bei der Einlösung der Beratungs- und Unterstützungsleistungen sollte ebenfalls das Wunsch- und Wahlrecht zur Anwendung kommen. Die Leistungen könnten bei der Selbsthilfe, den privaten oder ge- meinnützigen Trägern oder den Reha-Trägern von den Menschen mit Behinderungen abgerufen werden. Dies setzt allerdings voraus, dass die Reha- und Leistungsträger nach SGB IX die Chance einer personzentrierten Leistungserbringung erkennen, wenn Leistungen der Beratung und Unterstützung, wie sie beispielsweise im Persönlichen Budget formuliert sind, gewährt werden. Die Anerkennung und Finanzierung dieser Leistung wäre im Sinne der assistierten Selbstbestimmung und somit wäre ein Schritt in die Richtung der Umsetzung der UN-Behindertenrechtskonvention möglich.

\section{Das Persönliche Budget}

Mit der neuen Leistungsform, dem Persönlichen Budget, verfolgt der Gesetzgeber seit der Einführung des SGB IX Rehabilitation und Teilhabe im Jahre 2001 unterschiedliche Ziele. So sollen die Hilfen künftig individueller ausgestaltet und Teilhabeund Rehabilitationsbedarfe konkreter formuliert werden. Darüber hinaus geht es um die Stärkung der Selbstbestimmung und die Förderung der Eigenverantwortung von Menschen mit Behinderungen.

Wesentliches Ziel vor allem der Sozialhilfeträger ist es, mit der Bewilligung von Persönlichen Budgets ambulante Hilfen aus- und teil-stationäre und stationäre Hilfen abzubauen. In diesem Zusammenhang geht es um die Erschließung »neuer allgemeiner Dienstleistungen und Angebote «, die sich im bisherigen Angebotsspektrum für Menschen mit Behinderungen nicht etablieren konnten.

Einerseits wollen die Reha-Träger die Selbstbestimmung und befördern sie; andererseits kommt es immer wieder mit diesem im Sinne der Selbstbestimmung fortschrittlichen Instrument zu Problemen, beispielsweise beim Antragsverfahren, bei der Feststellung und Höhe der Bewilligung des Beratungs- und Unterstützungsbedarfes, bei Qualitätsvorgaben durch Reha-Träger und den damit verbundenen Vorstellungen zur Verwendung der Mittel oder bei der Auswahl des Personenkreises. Es stellt sich die Frage, ob Selbstbestimmung, die einen konkreten Geldbetrag nach sich zieht, möglicherweise doch nicht gewollt ist. Im Grunde steht hinter den Problembeispielen die Sorge der Reha-Träger, dass beispielsweise die bisherige Steuerung der Maßnahmen durch Zuweisungen in Einrichtungen nicht mehr möglich ist. Ein Warten auf RehaMaßnahmen und eine »Fallzahlensteuerung « mit Hilfe von » Wartelisten « dürfte es beim Einsatz von Persönlichen Budgets nicht mehr geben.

Was bewegt Menschen mit Behinderungen, Verbände, Dienstleister und den $\mathrm{Pa}$ ritätischen Wohlfahrtsverband die Umset- zung Persönlicher Budgets trotz zahlreicher Hemmnisse weiter zu befördern? Es ist die vom Gesetzgeber gewollte veränderte Rechtstellung der Menschen mit Behinderung. Sie können mit der neuen Leistungsform Selbstbestimmung und Wahlfreiheit praktizieren und erleben. Durch das Geld, das ihnen zur Verfügung steht, sind sie nicht mehr länger in der Rolle der Bittsteller.

Bundesweit liegen nach Kenntnis des Kompetenzzentrums Persönliches Budget des Paritätischen keine empirisch gesicherten Zahlen zur Anzahl Persönlicher Budgets in Deutschland vor. Nach Abschluss der Modellphase im Jahre 2007 konnten rund 845 evaluierte Persönliche Budgets festgestellt werden.

In der Antwort der Bundesregierung vom 6. Januar 2010 auf die Kleine Anfrage der Fraktion Die Linke wird betont, immer häufiger nutzten Menschen mit Behinderung das Persönliche Budget als Leistungsform. Die Bundesregierung verweist auf Zahlen, die von den Rehabilitationsträgern angegeben wurden. Demnach meldeten laut Bundesregierung die Sozialleistungsträger und Spitzenverbände 6.958 Persönliche Budgets im Dezember 2008. Davon wurden im Zeitraum vom 1. Januar bis zum 31. Dezember 2008 insgesamt 3.368 neue Persönliche Budgets gemeldet. Allerdings gibt die Bundesregierung zu bedenken, dass nicht alle Beteiligten den freiwilligen Meldungen nachgekommen sind und die Statistik daher unvollständig ist.

Darüber hinaus informiert die Regierung, dass sie derzeit prüfe, ob ein neues wissenschaftliches Vorhaben zum Persönlichen Budget sinnvoll ist. Diese Zahlen lassen sich sicherlich kritisch hinterfragen. Dabei kann festgestellt werden, dass es nach wie vor zu wenig Persönliche Budgets in Deutschland gibt. Die Bundestagsfraktion der Linken fordert in diesem Zusammenhang, die Bundesregierung solle sich mit den Verbänden zu den Problemen verständigen. Kritisch zu sehen ist allerdings die Aussage, dass das Persönliche Budget wirkungslos ist. Für Menschen, die ihre Hilfe im Rahmen des Persönlichen Budgets umsetzen, ist "Zufriedenheit « eine Wirkung, auch wenn die Anzahl dieser Personen natürlich unbefriedigend ist. Allerdings können wir beobachten, dass die Nachfrage zu den Beratungen im Kompetenzzentrum des Paritätischen Wohlfahrtsverband stetig steigt. Dies ist natürlich kein Beweis für die Steigerung der Anzahl Persönlicher Budgets - aber die starke Nachfrage lässt eine deutliche Steigerung der Zahlen vermuten.

\section{Wohnen, Teilhabe und Pflege}

Schon in der letzten Legislaturperiode, hatten die Freie Wohlfahrtspflege, der Deutsche Verein und die Interessenverbän- 
de behinderter Menschen im Rahmen der geplanten Weiterentwicklung der Eingliederungshilfe ein ganzheitliches Konzept bezüglich der Pflege und Eingliederungshilfe gefordert. Bis heute ist ein solches Konzept nicht in Sicht.

Die Ergebnisse des Modellprojekts zur Weiterentwicklung des Pflegebedürftigkeitsbegriffs sind grundsätzlich für alte pflegebedürftige Menschen positiv zu sehen, allerdings fehlen im Rahmen des Projekts verlässliche Zahlen zu den Kosten, die mit der Einführung eines neuen Assessment, eines erweiterten Begriffs für Pflegebedürftigkeit und in diesem Zusammenhang möglicherweise mit weiteren Leistungen verbunden sind.

Es bleibt offen, wer diese zusätzlichen Ausgaben übernehmen soll. Schon jetzt befürchten Sozialhilfeträger erhöhte Ausgaben für pflegebedürftige alte Menschen, wenn mit der Ausweitung der Leistungen keine bessere Ausstattung der finanziellen Mittel der Pflegeversicherung verbunden ist. Allerdings wird vonseiten der Sozialhilfeträger zugleich eine mögliche Entlastung bei den Leistungen der Eingliederungshilfe für Menschen mit Behinderungen gesehen. Sie gehen von Überschneidungen bei den Leistungen der Teilhabe und Pflege aus.

Die Verbände der Interessenvertretungen für Menschen mit Behinderungen und die Freie Wohlfahrtspflege betrachten diese Entwicklung mehr als kritisch. Der bereits mit der Einführung der Pflegeversicherung entstandene Streit zur Abgrenzung der Leistungen der Eingliederungshilfe zu den Leistungen der Pflege ist neu belebt. Neu ist allerdings, dass die Bundesregierung die UN-Behindertenrechtskonvention ratifiziert und anerkannt hat.

Die Behindertenrechtskonvention kennt den Begriff »Care « nicht. Die Konvention steht vielmehr für ein neues Verständnis von Behinderung, für assistierte Selbstbestimmung, Barrierefreiheit, Partizipation und der Forderung nach sozialer Inklusion. Sie konkretisiert fundamentale Menschenrechte und wird somit künftig die Grundlage für eine Sozialpolitik der »Rechte « bilden, in denen Teilhabe auch für Menschen mit einem hohen Unterstützungsbedarf in der Basis- und Körperpflege als Voraussetzung zur Teilhabe Selbstverständlichkeit sein wird. Demnach wäre es eine Menschenrechtsverletzung, wenn Menschen mit Behinderungen gegen ihren Willen ausschließlich Unterstützungsleistungen in einer Pflegeeinrichtung zugesprochen werden.

Solche "Verschiebungen " sind allerdings auch heute schon rechtswidrig, da sie das Wunsch- und Wahlrecht der Menschen ignorieren. Zu begrüßen ist, dass die Aktion Mensch das Projekt der LAG Ambulante Dienste in Hessen »Wohnen am richtigen Platz« fördert. Vom Träger werden häusli- cher Hilfen für Menschen mit Behinderung aus stationären Einrichtungen, insbesondere der Altenhilfe aufgebaut. Zu bedauern ist, dass es hierfür eigens ein Projekt geben muss. Wenn die Reha-Träger und hier insbesondere die Sozialhilfeträger fachliche und nicht fiskalische Verantwortung für die Teilhabe für Menschen mit Behinderungen wahrnehmen würden, wären solche Projekte überflüssig.

\section{Unterstützung zur Teilhabe am Arbeitsleben}

Im Jahr 2009 wurde die Leistung »Unterstützte Beschäftigung « zur verbesserten Teilhabe am Arbeitsleben neu eingeführt. Mit der Leistung wird es möglich, Menschen mit Behinderungen auf dem allgemeinen Arbeitsmarkt zu integrieren, in dem für sie Leistungen der innerbetriebliche Qualifizierung und der Berufsbegleitung gewährt werden. Für Menschen mit Behinderung ist die unterstützte Beschäftigung eine Chance, mit deren Hilfe Teilhabe außerhalb einer Werkstatt für Menschen mit Behinderungen möglich wird.

Dies war ein Schritt in die richtige Richtung. Allerdings wurden die Erwartungen der Menschen mit Behinderungen nur zum Teil erfüllt, da die unterstützte Beschäftigung auf bestimmte Reha-Träger insbesondere dem Integrationsamt und der Bundesagentur für Arbeit begrenzt bleibt. Interessenverbände und Vertreter der Bundesregierung haben unterschiedliche Auffassungen zu den Möglichkeiten von Unterstützungsleistungen für Menschen mit Behinderungen auf dem ersten allgemeinen Arbeitsmarkt. Während auf der einen Seite vom Bundesministerium für Arbeit und Soziales die Auffassung vertreten wird »Wer Beschäftigung auf dem allgemeinen Arbeitsmarkt will, muss in Arbeitsmarktkategorien denken " und es demnach keine Lösung sein kann, »behinderte Menschen für werkstattbedürftig zu erklären, um Geld verfügbar zu machen, mit dem sie dann außerhalb der Werkstatt beschäftigt werden ", wird vonseiten der Betroffenenvertretungen ausgeführt, dass Menschen mit Behinderungen deshalb zu Recht Unterstützung beispielsweise in einer Werkstatt genießen, weil ihnen aufgrund der Behinderung und unter den alleinigen Bedingungen des Arbeitsmarktes eine Teilhabe am Arbeitsleben auf diesem nicht möglich ist. Zankapfel hier sind die Mittel der Eingliederungshilfe nach SGB XII, die für Menschen mit Behinderungen in Werkstätten eingesetzt werden und eben nicht für die Teilhabe am Arbeitsleben auf dem ersten allgemeinen Arbeitsmarkt zur Verfügung stehen sollen. Es ist nach Auffassung der Bundesregierung eine Unterstützungsleistung, die ausschließlich an die Institution die Werkstatt gebunden ist und in der in diesem Zusammenhang auch Sozialversicherungsleistungen für Menschen mit Behinderungen bei der Teilhabe am Arbeitsleben in einer Werkstatt für Menschen mit Behinderung gewährt werden.

Die Überlegungen der Arbeits- und Sozialministerkonferenz in den Eckpunkten zur Weiterentwicklung der Eingliederungshilfe hinsichtlich der Leistungen der Eingliederungshilfe für eine Teilhabe am Arbeitsleben außerhalb von Werkstätten sind daher zu begrüßen.

Bis dahin bleiben für Menschen mit Behinderungen, die ihre Teilhabe am Arbeitsleben auf dem allgemeinen Arbeitsmarkt in Form eines Persönlichen Budgets realisieren, Fragen zur sozialen Absicherung offen. Die Anfang November 2007 von der Bundesagentur für Arbeit gestellten Fragen, insbesondere zur Kranken- und Rentenversicherung von Budgetnehmenden, blieben bisher unbeantwortet.

Erfreulicherweise gibt es auch positive Entwicklungen, beispielsweise gewährt trotz offener Fragen die Bundesagentur für Arbeit seit Mitte 2009 Sozialversicherungsleistungen auch im Rahmen des Persönlichen Budgets, in Rheinland-Pfalz gibt es das Budget für Arbeit und das Land Niedersachsen und die Stadt Hamburg befinden sich in der Vorbereitung von Projekten zum Persönlichen Budget für Arbeit. Diese Projekte sind dem Grunde nach zu begrüßen. Die Chance liegt in entsprechenden Unterstützungsleistungen und damit verbunden in einen für Menschen mit Behinderungen barrierefreien Zugang zum allgemeinen Arbeitsmarkt. Dies könnte auch in Form eines steuerfinanziertes Teilhabegeldes geschehen, an dessen Kosten sich auch der Bund beteiligt.

\section{Teilhabeplanung und persönliche Zukunftsplanung}

Wenn das Ziel ambulante Hilfen vor stationären Hilfen umgesetzt werden soll, brauchen Menschen mit Behinderungen und deren Angehörige komplexe Unterstützungsleistungen. Dies wird nur möglich sein, wenn ein Pool von professionellen, ehrenamtlichen und familiären Unterstützern gefunden, organisiert und aufrechterhalten wird. In den Sozialgesetzbüchern IX und XII sind die rechtlichen Grundlagen für Teilhabeplanung und Koordination geregelt. Die Erfahrungen des Paritätischen Wohlfahrtsverbandes in den letzten Jahre zeigen aber, dass es erhebliche Umsetzungsschwierigkeiten in den Ländern und Kommunen gibt. Dabei wird der Sinn der Regelungen im SGB IX, $\mathbb{S} \mathbb{S} 10$ Abs. 1 und 13 Abs. 2 Nr. 3 oder auch im SGB XII, $\mathbb{} 58$ seitens der Leistungsanbieter nicht infrage gestellt. 
Bundesweit kann festgestellt werden, dass eine Vielzahl von unterschiedlichen Verfahrensweisen und in diesen wiederum unterschiedliche Instrumente zur Planung von Teilhabeleistungen von den Rehabilitationsträgern angewendet werden. Das wird wohl bei den verschiedenen Rehabilitationsträgern auch künftig so bleiben.

Alle notwendigen Leistungen zur Realisierung der Teilhabe und zur Koordination der Leistungen müssen innerhalb der individuellen Teilhabeplanung Berücksichtigung finden. Dies setzt eine umfassende Auseinandersetzung mit der Person und ihrem persönlichen Lebenskonzept sowie ihren Ressourcen und ihren eigenen sozialen Netzwerken voraus.

Unabhängig von den Instrumentarien ist jedoch sicherzustellen, dass jedes Verfahren der Teilhabeplanung für den leistungsberechtigten Menschen transparent und nachvollziehbar, in einfacher Sprache und unter Beachtung des Wunsch- und Wahlrechtes stattfinden sollte. Dazu gehört die Beteiligung des Menschen mit Behinderung oder die Beteiligung von Personen des Vertrauens. Die persönliche Zukunftsplanung ist ein Instrument, das sich »aus entsprechenden nordamerikanischen Entwicklungen des 'person-centered planning a ableitet (vgl. O'Brien \& O'Brien 2000; O'Brien \& O'Brien 2002) und findet seit den 1990er Jahren im deutschsprachigen Bereich zunehmend Verbreitung. Persönliche Zukunftsplanung steht im Spannungsverhältnis zu allen Ansätzen einer individuellen Hilfeplanung und bezeichnet einen Ansatz, der den betreffenden Menschen als aktiv Teilhabenden in den Mittelpunkt stellt und ausgehend von seinen Visionen und Träumen konkrete nächste Schritte ableitet (vgl. Boban \& Hinz 1999; Boban \& Hinz 2004). Damit ist persönliche Zukunftsplanung subjektzentriert, kompetenzorientiert, dialogisch und außerhalb institutioneller Strukturen verortet (vgl. Hinz 2005)《.

Die persönliche Zukunftsplanung richtet sich in erster Linie an die Bürgerin und den Bürger sowie den Unterstützerkreis in deren Umfeld. Dabei werden besonders Personen des Vertrauens des Menschen mit Behinderung eingebunden. In dem Prozess wird auf seine persönlichen Ziele und seine Ressourcen und die Ressourcen in seinem Umfeld geschaut, um die persönlichen Ziele umzusetzen. Sie ermöglicht das Aufspüren und das Darstellen persönlicher und differenzierter Lebensentwürfe, die sich nicht an vorhandenen Institutionen orientieren. Sie ermöglicht aber auch ein Aufspüren von professionellen, ehrenamtlichen und familiären Unterstützern. Persönliche Zukunftsplanung ist ein erster und wichtiger Baustein, um das Leben außerhalb von Institutionen mit komplexen Unterstützungsleistungen zu ermöglichen. Ein Bünd- nis von Unterstützerinnen und Unterstützern aus dem Umfeld der Person wird gestärkt. Das können Freunde, Bekannte und Verwandte sein.

Die sozialen Dienstleistungen stehen in diesem Prozess vor neuen Herausforderungen. Aufgrund der Gewährung von stationären Hilfen sind bei den meisten Bewohnerinnen und Bewohnern von stationären Einrichtungen kaum noch Unterstützer im sozialen Umfeld vorhanden. Erst nach geraumer Zeit des Wohnens in der eigenen Wohnung lassen sich neue Nachbarschaften und Bekannte im Umfeld der Menschen mit Behinderung identifizieren, die mit professioneller Begleitung bereit sind, Unterstützungsleistungen zu übernehmen.

Die Beschlüsse der Arbeits- und Sozialministerkonferenz zur Weiterentwicklung der Eingliederungshilfe greifen den Gedanken der personzentrierten Leistungen und des bürgerschaftlichen Engagements auf. Für die Umsetzung personzentrierter Leistungen und für die gezielte Einbindung von professionellen und ehrenamtlichen Kräften wäre vorstellbar, dass persönliche $\mathrm{Zu}$ kunftsplanung Bestandteil der Teilhabeplanung im weitesten Sinne wird. Das setzt voraus, dass diese individuell über die Leistung der Beratung und Unterstützung, wie im $\mathbb{1 7}$ SGB IX verankert, als Leistungen berücksichtigt und entsprechend finanziert wird. Es setzt weiterhin voraus, dass für die Umsetzung der Methode entsprechend Moderatoren ausgebildet werden. Hierin könnte unter Einbindung aller bestehenden Ressourcen die tatsächliche Chance einer personzentrierten Unterstützungsplanung und Umsetzung unter Hinzuziehung weiterer Akteure unabhängig von und neben bereits bestehenden Angeboten liegen.

\section{Was zu tun ist}

1 Autonomie und Inklusion gehören zu- sammen: Auf der Basis dieses Grundsatzes sind Strukturen zu befördern, aufzubauen und finanziell abzusichern, die ausschließlich eine den Menschen mit Behinderung verpflichtende Leistung anbieten, dazu gehören Beratung und Unterstützung.

2 Autonomie und assistierte Selbstbe- stimmung setzen Befähigung voraus: Ein System, in dem die Dienstleistungen von der Angebotsstruktur geprägt waren, braucht ein Umdenken aller Beteiligten. Das bedeutet, dass sowohl für Leistungsberechtigte als auch für Leistungs- und Angebotsträger Möglichkeiten der Auseinandersetzung mit und Qualifikation für das Neue geschaffen werden, beispielsweise dem Instrument der persönlichen Zukunftsplanung.
3 Autonomie braucht Barrierefreiheit: • Barrierefreiheit lässt sich nicht auf das Absenken von Bordsteigen reduzieren. Sie ist mehr. Dazu gehören Unterstützungsleistungen der Beratung und Begleitung, die für alle Menschen unabhängig von der schwere und dem Ausmaß der Behinderung zugängig sind.

4 Teilhabe- und Zukunftsplanung: Planung haben in einer Sprache oder Kommunikationsform zu erfolgen, die einen aufrichtigen Dialog mit dem jeweiligen Menschen zulassen.

5. Inklusion braucht Bekennung: Ein Umdefinieren des Begriffs von Integration in Inklusion wird der Sache nicht gerecht. Inklusion als Leitgedanken kann regionale Sozialräume nur verändern und befördern, wenn Gemeinden und Kommunen Verantwortung für alle Bürgerinnen und Bürger übernehmen. Hierzu gehören die Öffentlichkeitsarbeit zur UN-Behindertenrechtskonvention, Transparenz in der Behindertenberichterstattung und Transparenz zu dem von der Bundesregierung zu erstellenden nationalen Aktionsplan oder den Landes- und kommunalen Plänen.

6. Inklusion und bürgerschaftliches En- gagement gehören zusammen: Die Einbindung des bürgerschaftlichen Engagements darf nicht zu einem Rückzug der Reha- und Leistungsträger bei der Finanzierung niedrigschwelliger Hilfen führen. Es ist zu investieren in eine Kultur der Bündnisse zwischen professionellen, ehrenamtlichen und familiären Unterstützerinnen und Unterstützer. So kann langfristig eine spürbare finanzielle Entlastung für die Sozialhilfe ermöglicht werden.

7. Personzentrierung und inklusiver So- zialraum bedingen einander: Personzentrierte Hilfen sind nur umsetzbar, wenn eine Veränderung auf der Strukturebene erfolgt. Unterstützungsleistungen sind unabhängig von der Institution zu gewähren.

\section{Internet-Hinweise}

http://www.destatis.de/jetspeed/portal/cms/S ites/destatis/Internet/DE/Presse/pm/Uebersicht/Sozialleistungen,templateld=renderPrint.psml

http://dip21.bundestag.de/dip21/btd/17/oo4/17 oo406.pdf

http://www.lagfad-hessen.de/projektbeschreibung.pdf

http://www.steuerzahler.de/Verschwendung/1224b474/index.html

http://www.inklusionspaedagogik.de/content/blogcategory/29/79/lang,de 
Dazu gehören Leistungen der Beratung und Begleitung.

Q Personzentrierung benötigt ein neues ๑ Verständnis von Behinderung: Die bisherige medizinisch und defizitorientierte Feststellung der Behinderung ist von einem biopsychosozialen Modell, in dem die Rolle der Wechselwirkungen anerkannt wird, (ICF) abzulösen.
9. Inklusion und Teilhabe am Arbeitsleben bedeutet, Verantwortung zu übernehmen: Es sind verbindliche Regelungen für die Berufsorientierung zwischen der Bundesagentur für Arbeit und den Schulträgern zu treffen.

10. Inklusion braucht Netzwerke und -Verständigung: Netzwerke und Kooperation auf der Ebene der Reha- und
Leistungsträger und der Ebene des Leistungsberechtigten sind Voraussetzung für eine dem Menschen verpflichtende Leistungserbringung.

11 Personzentrierung braucht Rückhalt: Leistungen der Teilhabe sind bedarfsdeckend und wie bisher gleichberechtigt oder vorrangig vor den Leistungen der Pflege zu gewähren.

\section{Wie weiter in der Eingliederungshilfe?}

Die Weiterentwicklung der Eingliederungshilfe war bereits Bestandteil des Koalitionsvertrags in der letzten Legislaturperiode. Aber erst im November 2008 gab es einen Beschluss der Arbeits- und Sozialministerkonferenz und ein Vorschlag zur Weiterentwicklung der Eingliederungshilfe. Der erste Prozess der Anhörung der Verbände zu den Vorschlägen der Bund-Länder-Arbeitsgruppe wurde im Sommer 2009 abgeschlossen. Im Ergebnis umfangreicher Diskussionen, Anhörungen und Stellungnahmen hat nun die Bund-Länder-Arbeitsgruppe der Arbeits- und Sozialministerkonferenz im November 2009 einige Eckpunkte für eine Reform der Eingliederungshilfe vorgelegt und die Regierung gebeten, die Reformvorschläge noch in dieser Legislaturperiode umzusetzen.

Der Beschluss macht deutlich, dass die Weiterentwicklung der Eingliederungshilfe ein wichtiger Teil des nationalen Aktionsplans zur Umsetzung der UN-Behindertenrechtskonvention werden soll. Die Bund-Länder-Arbeitsgruppe erkennt die wichtigen Impulse an, die von der Konvention ausgehen. Dazu gehören unabhängige Lebensführung und Einbeziehung in die Gemeinschaft. Inklusion als Leitidee soll zu einem Wandel in der Ausgestaltung sozialer Unterstützungsleistungen führen. Gewollt ist in Übereinstimmung mit den Verbänden, dass die Eingliederungshilfeleistungen künftig nicht nur in Sondersystemen und ausschließlich in Form von professionellen Hilfen erbracht werden, denn Sondersysteme erschweren den Zugang zu allgemeinen Systemen.

Bei der kritischen Auseinadersetzung mit den Eckpunkten lassen sich wiederum Chancen und mögliche negative Entwicklungen ausmachen. Grundsätzlich positiv zu bewerten sind u. a., dass es bei den Veränderungen

- nicht zu Verschlechterungen kommen soll,

- der offene Maßnahmekatalog der Eingliederungshilfe erhalten bleiben soll und

- ein berufliches Orientierungsverfahren implementiert werden soll.

Das Vergabeverfahren in der Eingliederungshilfe ist nicht mehr, wie ursprünglich vorgesehen, Bestandteil in den Eckpunkten zur geplanten Reform. Darüber hinaus sollen die Leistungen zur Teilhabe am Arbeitsleben in der Werkstatt künftig nicht nur an diese Organisationsform gebunden sein. All dies zusammengenommen wäre ein großer Schritt in Richtung personen- und nicht einrichtungsorientierter Leistungen. Dies erfordert Leistungen der Beratung und Unterstützung. Darüber hinaus sind Unterstützungsangebote und ein barrierefreie Zugang zu diesen notwendig. Die Veränderungen dürfen jedoch nicht dazu führen, dass Menschen mit einem hohen Unterstützungsbedarf ausgeschlossen werden. Auch für sie gelten die Leitgedanken der UN-Behindertenrechtskonvention von assistierter Selbstbestimmung, Inklusion und Teilhabe.

Anlass zur Sorge geben allerdings Formulierungen im Koalitionsvertrag für diese Legislaturperiode. Auf den letzten Seiten ist nachzulesen:

"Zusammen mit den kommunalen Spitzenverbänden werden wir nach Wegen suchen, Entlastungen für die Kommunen, z. B. Flexibilisierung von Standards und Gleichstellung bei gesamtstaatlichen Aufgaben, und Erweiterungen des kommunalen Handlungsspielraums zu identifizieren.«

"Die Folgen der Wirtschafts- und Finanzkrise haben die Leistungsfähigkeit vieler Kommunen strapaziert und Fragen nach der Güte kommunaler Leistungsfähigkeit a ufgeworfen. Wir beabsichtigen, den Ländern vorzuschlagen, eine gemeinsame Bestandsaufnahme zu erarbeiten und Handlungsempfehlungen zur Stärkung der kommunalen Selbstverwaltung vorzulegen. Dabei sind auch Fragen der Finanzbeziehungen zwischen Bund, Ländern und Gemeinden (Konnexitätsprinzip) und der Beteiligung der Kommunen an der Gesetzgebung des Bundes einzubeziehen ..."

Was können diese Formulierungen für Leistungen der Eingliederungshilfe für Menschen mit Behinderungen bedeuten, etwa wie die Entwicklungen im Dezember 2009 in Schleswig Holstein. Oder wird es möglicherweise einen Gesetzesvorschlag wie das "Kommunale Entlastungsgesetz" (KEG) im Jahr 2005 geben. Seinerzeit war geplant die Kommunen u. a. dadurch zu entlasten, dass die Bedarfsdeckung nicht mehr Bestandteil der Sozialhilfe sein sollte. Oder liegt darin möglicherweise in diesen Formulierungen die Chance für ein Leistungsgesetz für Menschen mit Behinderungen, an dem sich der Bund beteiligt? Das erscheint in Anbetracht dessen, dass dazu keine Ausführungen im Beschluss der Arbeits- und Sozialministerkonferenz und in den Eckpunkten zur Weiterentwicklung der Eingliederungshilfe zu finden sind, eher unwahrscheinlich.

Der Umbau der Leistungen der Eingliederungshilfe ist also noch in dieser Legislaturperiode geplant. Unabhängig davon welchen Weg die Bundesregierung und die Länder einschlagen werden: Die Veränderungen werden sich an den Leitgedanken der Behindertenrechtskonvention der Vereinten Nationen messen lassen müssen. Hierzu gibt es eben auch eine Aussage im Koalitionsvertrag und darin liegt die Chance für Veränderungen im Sinne der Menschen mit Behinderungen, die von den Verbänden der Freien Wohlfahrtspflege und den Interessenverbänden behinderter Menschen grundsätzlich begrüßt werden.

Claudia Zinke 